$0.9, p=0.04)$. Blacks who were inactive, and insufficiently active Hispanics had a lower chance of good/excellent diabetes control $(\mathrm{AOR}=0.5,95 \% \mathrm{CI}=0.2-0.9, \mathrm{p}=0.03 ; \mathrm{AOR}=0.3,95 \% \mathrm{CI}=0.1-$ $0.7, \mathrm{p}=0.007$ respectively). DISCUSSION/SIGNIFICANCE OF IMPACT: The results show the importance of insurance coverage, food security and physical activity in diabetes control among different racial/ethnic groups. They indicate a need for affordable health care and for culturally-relevant interventions that include physical activity and food security.

Renal Tubular Complement C9 Deposition is Associated with Renal Tubular Damage and Fibrosis in Lupus Nephritis

Shudan Wang ${ }^{1}$, Ming Wu, MD², Luis Chiriboga, $\mathrm{PHD}^{2}$, Chaim

Putterman, $\mathrm{MD}^{1}$, Anna Broder, MD, MSc ${ }^{3}$, and H. Michael Belmont, $M D^{2}$

${ }^{1}$ Albert Einstein College of Medicine; ${ }^{2} \mathrm{NYU}$ Langone Health;

${ }^{3}$ Montefiore / Albert Einstein College of Medicine

OBJECTIVES/GOALS: Tubulointerstitial damage in lupus nephritis (LN) is a strong predictor of progression to chronic kidney disease and end stage renal disease (ESRD). While complement activation mediates glomerular injury, the role of complement in renal tubular damage has not been evaluated. We investigated the association between complement activation and tubulointerstitial fibrosis. METHODS/STUDY POPULATION: Patients with LN were selected randomly between July 2014 - July 2016. Chromogenic immunohistochemistry was performed on formalin-fixed, paraffin-embedded, $4-\mu \mathrm{m}$ human renal biopsy sections using unconjugated, murine anti-human Complement C9 (Hycult Biotech, clone X197) as a marker of the terminal complement activation. Positive control is C3 glomerulopathy and negative control is normal kidney. Tubular basement membrane C9 staining intensity were analyzed on semiquantitative scale 0 to 3 by a renal pathologist. Interstitial fibrosis/tubular atrophy were categorized into low (0-10\%), medium (11-20\%), or high ( $\geq 21 \%)$. Clinical parameters were assessed at time of biopsy and 6 months post biopsy. Bivariate associations were assessed between presence of tubular $\mathrm{C} 9(\mathrm{C} 9+)$ and other covariates. RESULTS/ANTICIPATED RESULTS: Renal biopsies from $30 \mathrm{LN}$ studied, $23(77 \%)$ of which had proliferative LN. There were 24 (80\%) women, mean (SD) age 33 (12) years. Positive tubular C9 staining was observed in 7/30 (23\%) biopsies. At time of renal biopsy, C9+ patients had significantly higher urine protein, compared to C9- patients: median (IQR) 6.2g (3.3-13.1) vs. 2.4g (1.3-4.6), $\mathrm{p}<0.01$. The differences persisted at 6 months after induction therapy: $1.08 \mathrm{~g}(1.0-8.3)$ in C9+ vs. $0.68 \mathrm{~g}(0.2-2.1)$ in C9- patients, $\mathrm{p}=0.06$. There was no significant difference in creatinine at renal biopsy between the two groups. Tubular C9 deposition was associated with interstitial fibrosis: $49 \%$ had severe interstitial fibrosis vs. none in the C9- group, $\mathrm{p}=<0.01$. Higher proportion of $\mathrm{C} 9+$ patients had moderate NIH Chronicity index: $42.9 \%$ vs $8.7 \%$ in the C9- group, $p=0.07$. DISCUSSION/SIGNIFICANCE OF IMPACT: Tubular C9 deposition is significantly associated with proteinuria, interstitial fibrosis and increased chronicity which predict progression to ESRD and high mortality. This finding suggests that complement activation in the tubules may be linked to proteinuria and contribute to mechanism in tubulointerstitial damage in LN.

\section{Risk Aversion in Lung Transplantation: Organ Procurement Organizations Differ in Willingness to Pursue Non-ideal Donor Organs}

Samantha Ellen Halpern ${ }^{1}$, Alec McConnell, Sarah Peskoe, Vignesh Raman, Oliver K Jawitz, Ashley Y Choi, John C Haney, Jacob A Klapper, and Matthew G Hartwig

${ }^{1}$ Duke University

OBJECTIVES/GOALS: Lung transplant (LTx) candidates benefit from use of non-ideal donor organs. Each organ procurement organization (OPO) defines "acceptable" donor organs introducing unmeasured variation in donor pursuit. We characterized non-ideal donor pursuit among OPOs to identify drivers of risk aversion in LTx. METHODS/STUDY POPULATION: We queried the UNOS registry for adult donors who donated $\geq 1$ organ for transplantation from 12/2007-12/2018. Non-ideal donors were those with any of age $>50$, smoking history $\geq 20$ pack-years, $\mathrm{PaO}_{2} / \mathrm{FiO}_{2} \quad(\mathrm{P} / \mathrm{F})$ ratio $<350$, donation after cardiac death (DCD) status, or $\mathrm{CDC}$ increased risk (IRD) status. Non-ideal donor pursuit rate was defined as the proportion of non-ideal donors at each OPO from whom consent for lung donation was requested with lower numbers indicating increased risk aversion. We estimated the correlation between nonideal and overall donor pursuit using a Spearman correlation coefficient. Adjusted non-ideal donor pursuit rates were estimated using multivariable logistic regression. RESULTS/ANTICIPATED RESULTS: Overall, 18,333 deceased donors were included and classified as ideal or non-ideal. Among 58 OPOs, rates of non-ideal donor pursuit ranged from 0.24-1.00 Figure). Of 5 non-ideal characteristics, DCD and IRD status were associated with the most and least risk aversion, respectively. Non-ideal donor pursuit was strongly correlated with overall donor pursuit $(\mathrm{r}=0.99)$. On adjusted analysis, older age (OR 0.15, 95\% CI 0.13-0.16), smoking history (OR $0.38,95 \%$ CI $0.34-0.44$ ), low $\mathrm{P} / \mathrm{F}$ ratio (OR 0.12 , 95\% CI 0.11-0.14), and DCD status (OR 0.04, 95\% CI 0.03-0.04) were all independently associated with significant risk aversion, corresponding to decreased rates of donor pursuit. DISCUSSION/SIGNIFICANCE OF IMPACT: OPOs differ in their levels of risk aversion in LTx and risk aversion is not uniform across selected categories of non-ideal lung donor. Consideration of new OPO performance metrics that encourage the pursuit of non-ideal lung donors is warranted.

\section{6}

Sex Differences in the Cortical Structure in Children with Irritability and Disruptive Behavior ${ }^{\dagger}$

Karim Ibrahim ${ }^{1}$, Fangyong $\mathrm{Li}^{1}$, George $\mathrm{He}^{2}$, Kevin Pelphrey ${ }^{3}$, Gregory McCarthy ${ }^{2}$, and Denis Sukhodolsky ${ }^{1}$

${ }^{1}$ Yale University School of Medicine; ${ }^{2}$ Yale University; ${ }^{3}$ University of Virginia

OBJECTIVES/GOALS: This study examines sex differences in brain structure in youths with disruptive behavior disorders (DBD). We use measures of gray matter volume (GMV) in regions-of-interest implicated in the pathophysiology of conduct problems and a whole-brain analysis of cortical thickness. We also examine unique associations between brain structure and callous-unemotional traits. METHODS/STUDY POPULATION: This study included 90 children with a DBD (30 females) aged 8-16 and 50 Healthy Controls 
(20 females) matched for age and IQ. Children received a diagnostic evaluation using the K-SADS. Pre-processing was conducted using FreeSurfer. For ROI analyses, multivariate GLM models were conducted in SPSS for estimates of GMV to examine the main effects of diagnosis and sex, and sex-by-diagnosis interactions. Whole-brain analyses were conducted in FreeSurfer. Associations were examined between structure and parent ratings of callous-unemotional (CU) traits using the Inventory of Callous-Unemotional Traits in regression analyses in the DBD group, while controlling for the variance in aggressive behavior using the Child Behavior Checklist Aggressive Behavior Scale. All analyses controlled for differences in intracranial volume. RESULTS/ANTICIPATED RESULTS: Relative to controls, children with DBD showed reduced GMV in the bilateral amygdala (left: $p=.004$; right: $p=.04$ ). Sex-by-diagnosis interactions were observed in the left ventromedial prefrontal cortex $(p=.004)$, right insula $(p=.001)$, right inferior frontal gyrus $(p=.02)$, and bilateral anterior cingulate (left: $p=.02$; right: $p=.01$ ) in which DBD males showed lower and DBD females showed higher GMV relative to respective controls. For whole-brain analyses, a significant sex-bydiagnosis interaction was observed in the left ventromedial prefrontal cortex and supramarginal gyrus indicating that DBD males showed lower and DBD females showed higher cortical thickness relative to respective controls. Sex-by-CU traits interactions were observed for left amygdala and ACC volumes. DISCUSSION/ SIGNIFICANCE OF IMPACT: The current study provides evidence of reduced amygdala volume in children with $\mathrm{DBD}$, and interactions between sex and diagnosis in the ventromedial prefrontal cortex and supramarginal gyrus, which may have implications for identifying sex-sensitive neural biomarkers. CONFLICT OF INTEREST DESCRIPTION: Disclosures: Dr. Sukhodolsky receives royalties from Guilford Press for a treatment manual on CBT for anger and aggression in children. Drs. Ibrahim, He, Pelphrey, McCarthy, and Mr. Li have no biomedical financial interests or potential conflicts of interest to declare related to this present study.

\section{2}

Special Delivery: Home Delivery of Healthy Food to Young Women during Pregnancy*

lone Locher, Medical Student ${ }^{1}$, Marika Waselewski, and Tammy Chang

${ }^{1}$ University of Michigan School of Medicine

OBJECTIVES/GOALS: Most pregnant youth (ages 14-24) gain more weight during pregnancy than recommended by clinical guidelines. We aim to describe the feasibility and acceptability of home grocery delivery of fruits, vegetables, and healthy snacks to promote healthy weight gain in this vulnerable population. METHODS/STUDY POPULATION: Participants were low-income pregnant youth in Michigan. Each participant was sent biweekly grocery deliveries consisting of $\$ 35$ worth of fresh fruits, vegetables, and healthy snacks via the app-based delivery service, Shipt. Between deliveries, participants were prompted to respond to weekly text message-based surveys of a 24-hour food recall. This validated nutritional assessment quantifies consumption of fruit and vegetable servings. In addition, participants were asked to send daily photos and descriptions of foods they were eating. This study was approved by the University of Michigan Institutional Review Board. RESULTS/ANTICIPATED RESULTS: To date, 27 participants have been enrolled. Thirteen participants have completed their participation, 4.3 months on average, and were sent an average of 10 grocery deliveries each. In total, over 200 deliveries have been sent with $86 \%$ confirmed by the study participant (179/207). Additional outcomes to be assessed include: 1) text message response rates by participants and 2) content from photos and text descriptions of food eaten by participants. The 24-hour recall and text and photo messaging provided in-context data about grocery utilization. DISCUSSION/SIGNIFICANCE OF IMPACT: Grocery delivery is both feasible and acceptable to our youth participants. Use of grocery delivery constitutes a novel intervention to promote healthy weight gain in pregnancy for vulnerable populations through improving access to healthy food options.

4145

\section{Stepping Stones for Success in T3-T4 Translation: Building Collective Impact}

Kishore Athreya ${ }^{1}$, Terry Nakazono ${ }^{2}$, Jim Morrison ${ }^{2}$, and Pamela Davidson ${ }^{2}$

${ }^{1}$ David Geffen School of Medicine at UCLA; ${ }^{2}$ UCLA Clinical and Translational Science Institute

OBJECTIVES/GOALS: We will investigate the influence of multisector partnerships in T3-T4 research associated with advances in delivery systems, patient/population outcomes and health policy and the translational processes linked to these improvements. METHODS/ STUDY POPULATION: We are using both quantitative and qualitative data to measure and analyze partnership characteristics linked to successful translation into practice \& policy. We aim to complete 100 surveys of investigators who have conducted CTSA-supported T3-T4 research to examine partnerships, conditions of collective impact, and quantifiable changes in delivery systems, health outcomes, and policy. Using rigorous criteria, we will select projects for more in-depth interviews to understand the practices of successful translation and roadblocks and barriers that challenge translation. RESULTS/ANTICIPATED RESULTS: The anticipated research products include: (i) an analytic report on partnership structure and processes and the statistical associations to stages of change outcomes, (ii) a series of vignettes to describe the impact stories and translational processes, (iii) cross-project analysis of the data and vignettes to produce generalizable information to improve T3T4 translation, and (iv) peer-reviewed manuscript(s) for publication. DISCUSSION/SIGNIFICANCE OF IMPACT: The study will inform and improve researcher competencies and accelerate translation in CTSA hubs that emphasize T3-T4 research. We will develop novel definitions of the T3-T4 research impact. Ultimately, the results will inform research training to better address real-world priorities and needs.

Stromelysin-1 as a biomarker for acute lung injury

Andrea Sikora Newsome ${ }^{1}$, Rana Kadry, Sandeep Arthram, Timothy Jones, and Somanath Shenoy

${ }^{1}$ University of Georgia

OBJECTIVES/GOALS: Acute lung Injury (ALI) has long been considered a proceeding event to the development of Acute Respiratory Distress Syndrome (ARDS). Diagnosis of classical ALI and ARDS remains difficult relies on clinical components of the Berlin Criteria, interpretation of radiographs and exclusion of pulmonary 